\title{
Expanding the Computational Anatomy Gateway from clinical imaging to basic neuroscience research
}

\author{
Daniel Tward \\ dtward@cis.jhu.edu \\ Johns Hopkins University, Center for \\ Imaging Science \\ Baltimore, Maryland
}

\author{
Anthony Kolasny \\ Johns Hopkins University, Center for \\ Imaging Science \\ Baltimore, Maryland
}

\author{
Fatima Khan \\ Johns Hopkins University, Center for \\ Imaging Science \\ Baltimore, Maryland
}

\author{
Juan Troncoso \\ The Johns Hopkins University School \\ of Medicine, Department of Pathology \\ Baltimore, Maryland
}

\author{
Michael Miller \\ Johns Hopkins University, \\ Department of Biomedical \\ Engineering \\ Baltimore, Maryland
}
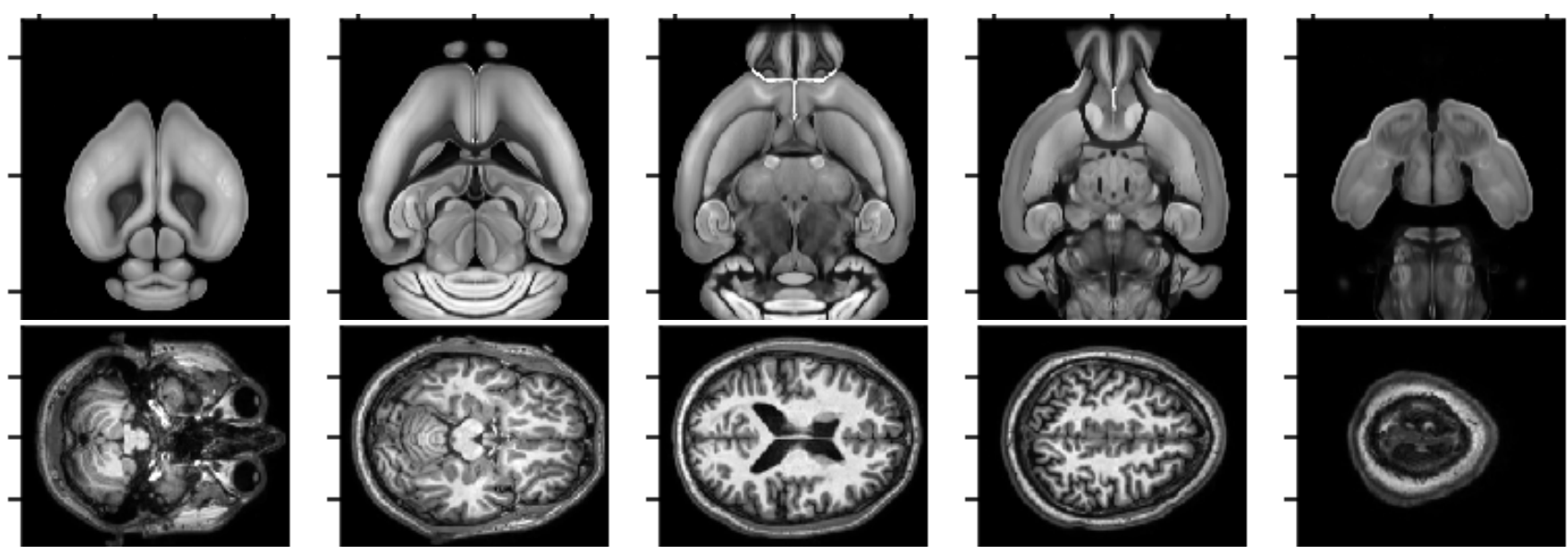

Figure 1: Brain mapping using the Allen Institute's common coordinate framework atlas image is a critical tool for neuroscientists working with mouse models (top), as are human MRI atlases (bottom) already used by the computational anatomy gateway.

\begin{abstract}
The Computational Anatomy Gateway, powered largely by the Comet (San Diego Supercomputer Center) and Stampede (Texas Advanced Computing Center) clusters through XSEDE, provides software as a service tools for atlas based analysis of human brain magnetic resonance images. This includes deformable registration, automatic labeling of tissue types, and morphometric analysis. Our goal is to extend these services to the broader neuroscience community, accommodating multiple model organisms and imaging modalities, as well as low quality or missing data. We developed

Permission to make digital or hard copies of all or part of this work for personal or classroom use is granted without fee provided that copies are not made or distributed for profit or commercial advantage and that copies bear this notice and the full citation on the first page. Copyrights for components of this work owned by others than ACM must be honored. Abstracting with credit is permitted. To copy otherwise, or republish, to post on servers or to redistribute to lists, requires prior specific permission and/or a fee. Request permissions from permissions@acm.org.

PEARC '19, fuly 28-August 1, 2019, Chicago, IL, USA

(C) 2019 Association for Computing Machinery.

ACM ISBN 978-1-4503-7227-5/19/07 ..\$15.00

https://doi.org/10.1145/3332186.3332217
\end{abstract}

a new approach to multimodality registration: by predicting one modality from another, we can replace ad hoc image similarity metrics (such as mutual information or normalized cross correlation) with a log likelihood under a noise model. This statistical approach enables us to account for missing data using the Expectation Maximization algorithm. For portability and scalability we have implemented this algorithm in tensorflow. For accessibility we have compiled and many working examples for multiple model organisms, imaging systems, and missing tissue or image anomaly situations. These examples are made easily usable in the form of Jupyter notebooks, and made publicly available through github. This framework will significantly reduce the barrier to entry for basic neuroscientists, enabling the community to benefit from atlas based computational image analysis techniques.

\section{CCS CONCEPTS}

- Applied computing $\rightarrow$ Imaging; Computational biology; • Software and its engineering $\rightarrow$ Software usability. 


\section{KEYWORDS}

neuroimaging, brain mapping, image registration

\section{ACM Reference Format:}

Daniel Tward, Anthony Kolasny, Fatima Khan, Juan Troncoso, and Michael Miller. 2019. Expanding the Computational Anatomy Gateway from clinical imaging to basic neuroscience research. In Practice and Experience in Advanced Research Computing (PEARC '19), July 28-August 1, 2019, Chicago, IL, USA. ACM, New York, NY, USA, 6 pages. https://doi.org/10.1145/3332186. 3332217

\section{INTRODUCTION}

The field of neuroimaging informatics [14] is building a quantitative understanding of neuroimages and is poised to revolutionize neuroradiology and psychiatry. The computational burden at the heart of this field is a mapping engine, for nonrigidly registering observed neuroimages to families of well characterized atlases [2]. These mappings from large populations of neuroimages to a common space allows for a statistical characterization of neuroanatomy, including neurodegenerative disease. The Computational Anatomy Gateway (www.mricloud.org) provides web based software for this analysis of human brain magnetic resonance images (MRIs), and is powered largely by the Comet (San Diego Supercomputer Center) and Stampede (Texas Advanced Computing Center) clusters through XSEDE. These related services include automatic registration and segmentation of brain MRI, and statistical shape analysis of subcortical grey matter structures [9].

The benefit of these techniques are not limited human anatomy. Atlas based neuroimage analysis is proving increasingly critical throughout neuroscience [26], as large consortiums such as the BRAIN Initiative Cell Census Network are publishing data in common coordinates such as the Allen Reference Atlas [6]. Our goal is to expand the Computational Anatomy Gateway services to the larger neuroscience community, accommodating multiple model organisms and multiple imaging modalities. Outside the well controlled setting of human clinical MRI, many challenges still remain before high high quality analysis can be offered as a service. We subdivide these into challenges related to algorithms, computation, and accessibility, which we discuss below. All our code is open source and available via https://github.com/dtward/image_lddmm tensorflow/.

\section{RESULTS}

\subsection{Algorithm}

Two challenges we have identified as critical are the problem of accurate image registration between different modalities, and missing data or artifacts in images. We have recently developed a novel method to tackle these jointly [23]. Rather than building complex image similarity metrics to compare images from different modalities (such as mutual information [27] or normalized cross correlation [1]), we predict one modality from the other, transforming image intensities using polynomial regression. Given this prediction, image similarity can be computed using sum of square error, or log likelihood under a Gaussian white noise model. This statistical interpretation allows us to work with missing data and artifacts in using a classical Expectation Maximization (EM) algorithm [5].
Image registration is posed as an optimization problem. Given an atlas image $I: X \subset \mathbb{R}^{3} \rightarrow \mathbb{R}$, an observed image $J: X \rightarrow \mathbb{R}$, an unknown spatial transformation (registration) $\varphi: X \mapsto X$ which acts on the atlas image as $\varphi \cdot I=I\left(\varphi^{-1}\right)$, and an unknown polynomial transformation $F_{\theta}(t)=\sum_{i=0}^{N} \theta_{i} t^{i}$, we wish to estimate $\varphi, \theta$ by minimizing the weighted sum of square error

$$
\arg \min _{\varphi, \theta} \frac{1}{2 \sigma_{R}^{2}} R(\varphi)+\frac{1}{2 \sigma_{M}^{2}} \int\left|F_{\theta}\left(I\left(\varphi^{-1}(x)\right)\right)-J(x)\right|^{2} W(x) d x .
$$

The transformation $\varphi$ consist of a deformation which is generated by integrating a time varying velocity field, and a 12 parameter affine (linear and translation) transformation. The regularization function $R$ is an integral over time of its kinetic energy. This Large Deformation Diffeomorphic Metric Mapping (LDDMM) approach is described in detail elsewhere [2]. The constants $\sigma_{R}$ and $\sigma_{M}$ control the relative importance of Regularization and Matching accuracy terms.

$W$ is a spatially varying weight, equal to the posterior probability that the pixel at the location $x$ corresponds to some point in the atlas. This is computed using a Gaussian mixture model approach. We allow three possible labels for each pixel, estimating their mean from the data, and specifying their variance through three parameters: $\sigma_{M}^{2}$ (for matching to the atlas) $\sigma_{A}^{2}$ (for artifact) and $\sigma_{B}^{2}$ (for background, or missing tissue).

The algorithm proceeds as follows

(1) Choose an initial guess for $\varphi$ and $\theta$

(2) E step: Calculate the posterior probability $W$ that each observed voxel belongs to the atlas

(3) M step: Calculate optimal $\theta$ by weighted least squares, calculate optimal $\varphi$ using weighted LDDMM.

(4) Repeat steps 2-3 until convergence.

We note that this approach extends easily to mappings between images of arbitrary dimension (e.g. grayscale to grascale, grayscale to RGB, RGB to RGB), see reference for details [23].

\subsection{Computation}

To implement this algorithm numerically, all images and deformation fields are discretized in a regular 3D array. Deformations are applied to images using trilinear interpolation. The LDDMM framework builds transformations by integrating a time varying velocity field, which is discretized into 10 timesteps and integrated using the method of characteristics [19]. The computational complexity of these steps has been discussed in previous work [24, 25] and is dominated by linear interpolation and fast Fourier transforms. Transformations $\varphi$ are estimated iteratively by gradient descent, and intensity transformation parameters $\theta$ are estimated by weighted least squares at each iteration. Figure 2 shows an example of parameters being estimated as a function of iterations of gradient descent for the example https://github.com/dtward/image_lddmm_ tensorflow/blob/master/command_line_example.sh, demonstrating that estimates are beginning to converge in about 200 iterations.

We have chosen tensorflow (tensorflow.org) as a platform for high performance computing due to its wide support and community, as well as its portability between CPUs, GPUs, and other parallelization approaches. We have investigated running this tool on both CPU and GPU platforms, which the tensorflow backend 

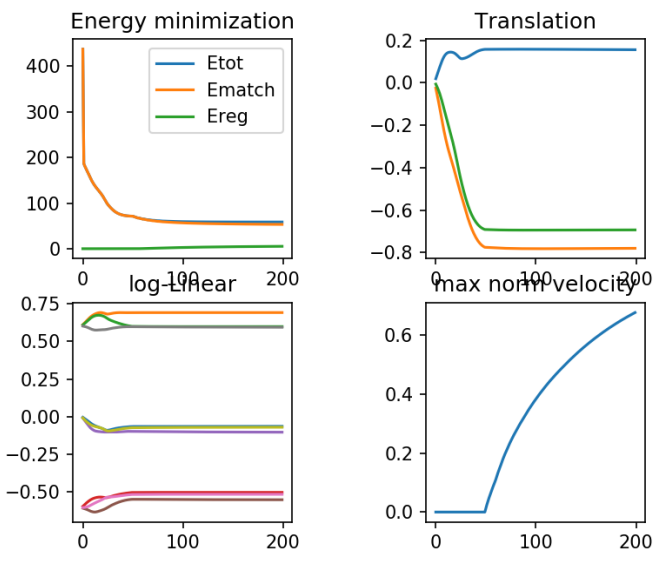

Figure 2: Parameters being estimated in a typical run of our registration algorithm as a function of gradient descent iterations. Top left: Cost function being minimized including total cost, matching accuracy, and regularization. Top right: 3 components of translation vector. Bottom left: 9 components of linear transformation (plotted after taking the matrix log). Bottom right: maximum of the velocity field generating the deformation.

allows without any change to code. The example in Fig. 2 above, deforming a $160 \times 264 \times 228$ voxel image, takes 18 minutes on a NVIDIA Titan RTX with $24 \mathrm{~GB}$, roughly a $4-5$ times speedup over a 16 core CPU. We also successfully tested it on XSEDE resources through Stampede using the Intel CPU environment, and are investigating performance on Comet using the CUDA GPU environment through singularity. For an image of size $Q \times R \times S$, the complexity of this algorithm is order $O(Q R S \log (Q R S))$, owing to a 3D Fast Fourier Transform involved in the regularization function. In practice however, the algorithm is dominated by trilinear interpolation, which is order $O(Q R S)$. Typically run times scale linearly with image size in this way.

\subsection{Accessibility}

To make these tools accessible to the broad neuroscience community we require an interface that does not require coding knowledge, and we require a set of well documented examples that are applicable to a wide variety of researchers. To achieve this we have presented image registration workflows through publicly available Jupyter notebooks (jupyter.org). Each notebook covers a different model organism or imaging modality. Users are invited to download these notebooks to reproduce results, or to find an example that closely matches their data, and simply replace filenames with their own. One example notebook is shown in Fig. 3. All the parameters required for an accurate registration for this image modality and model organism have been carefully chosen, reducing the barrier to entry for basic neuroscientists not familiar with image registration algorithms. For interactivity, visualization is presented and updated in real time as the algorithm progresses. Users can visualize their deforming atlas images, current estimates of the presence of artifacts or missing tissue, and other updates to verify that the algorithm is working as expected before committing to large batch jobs.

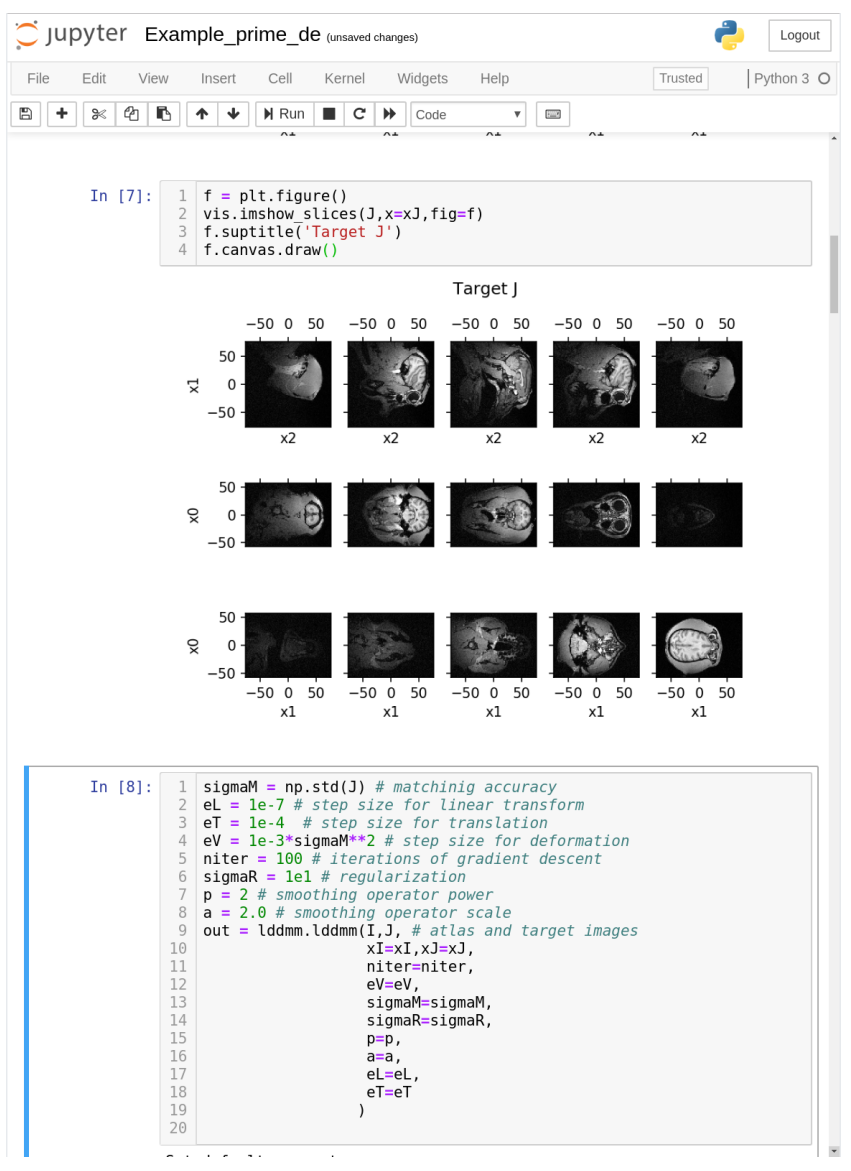

Figure 3: Example Jupyter notebook interface while registering a monkey dataset.

In Fig. 4 we show an example alignment for mouse data, between the Allen Common Coordinate Framework atlas [12] and a serial two photon microscopy image associated with a typical tracing experiment. In this experiment, a viral fluorescent label is injected into the brain, leading to a bright artifact. Using traditional image registration methods, this artifact will reduce mapping accuracy in the location where it is most important. Our method detects the artifact and is able to produce an accurate mapping, even with a grossly different image intensity profile.

Available atlases for several other model organisms are shown in Fig. 5. This data includes a human atlas from the MRI Cloud [15], the Waxholm rat atlas [16], a primate atlas from primate data exchange [13], and a cat atlas from the Cerebral Systems Lab [20]. We are continuing to grow our list of model organisms and image modalities by reviewing publicly available databases, improving access for neuroscientists in many different subfields. 


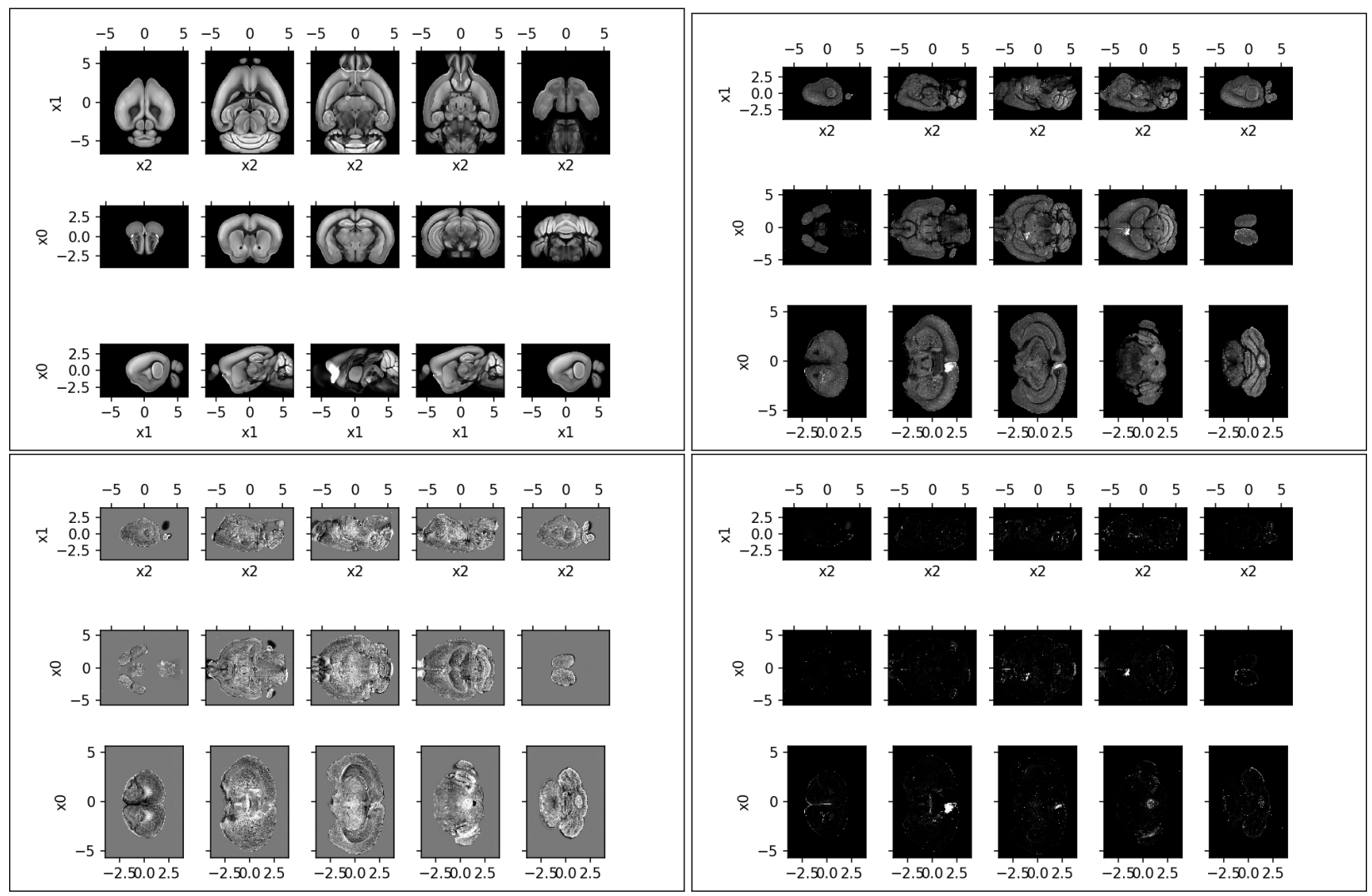

Figure 4: Top left to bottom right: Allen Common Coordinate framework atlas $I$, serial two photon image with injection site artifact $J$, error after registration $F_{\theta}\left(I\left(\varphi^{-1}\right)-J\right)$ (gray means low error), artifact detection $W$.

\section{DISCUSSION}

Image registration tools have become quite well established in human MRI analysis with many available options [10]. Our choice of the LDDMM framework is based upon (1) mathematical guarantees that transformations will always be smooth and invertible [7], (2) on the ability to work with sparse representations to address the bias variance tradeoff in modeling and statistical testing [21], and (3) on favorable properties for estimating local volume changes [22] which is critical for common neuroscience applications that investigate neuron density. In other areas of brain imaging the technology is less mature, and the data is more heterogeneous. It is common for groups to perform registration manually, by placing hundreds or even thousands of landmarks such as in the MouseLight project [8] An exciting new experimental paradaigm involves cleared brain tissue, with methods such as iDISCO [18] or CLARITY [3, 4]. Some registration algorithms have been designed for specific cleared tissue modalities such as ClearMap [17], or NDREG [11]. Rather than re-purposing tools from medical imaging, or designing algorithms of narrow applicability, the approach described here was specifically designed accommodate a wide variety of image modalities used in neuroimaging experiments, and be robust to artifacts and missing or damaged tissue common in these settings.

There are several limitations to our choice of working with tensorflow. For example, at the time of writing this manuscript tensorflow does not have built in 3D interpolation, which forced us to create our own python implementation with a likely performance cost. At the time the project was built tensorflow did not support FFTs with the complex128 data type which forced us into a lower precision. This has since been rectified. Because future changes to tensorflow are uncertain, we are currently building these tools to support several different backends, including PyTorch (pytorch.org), pure NumPy (numpy.org), and Simple ITK (simpleitk.org).

As this technology and examples become more polished, we plan to incorporate it into the Computational Anatomy Gateway, offering it through a software as a service / cloud computing model, in addition to this downloadable code. Our choice of tensorflow as an implementation will allow us to exploit high performance computing resources including GPU without rewriting any code. By overcoming challenges associated to heterogeneous neuroimaging data, we are able to expand our services and make atlas based image registration and analysis accessible to the broad neuroscience community.

\section{ACKNOWLEDGMENTS}

This work was supported by the National Institutes of Health (NIH) (www.nih.gov) grants P41EB015909, R01NS086888, R01EB020062, R01NS102670, U19AG033655, R01MH105660; the National Science Foundation (NSF) (www.nsf.gov) 16-569 NeuroNex contract 1707298; 

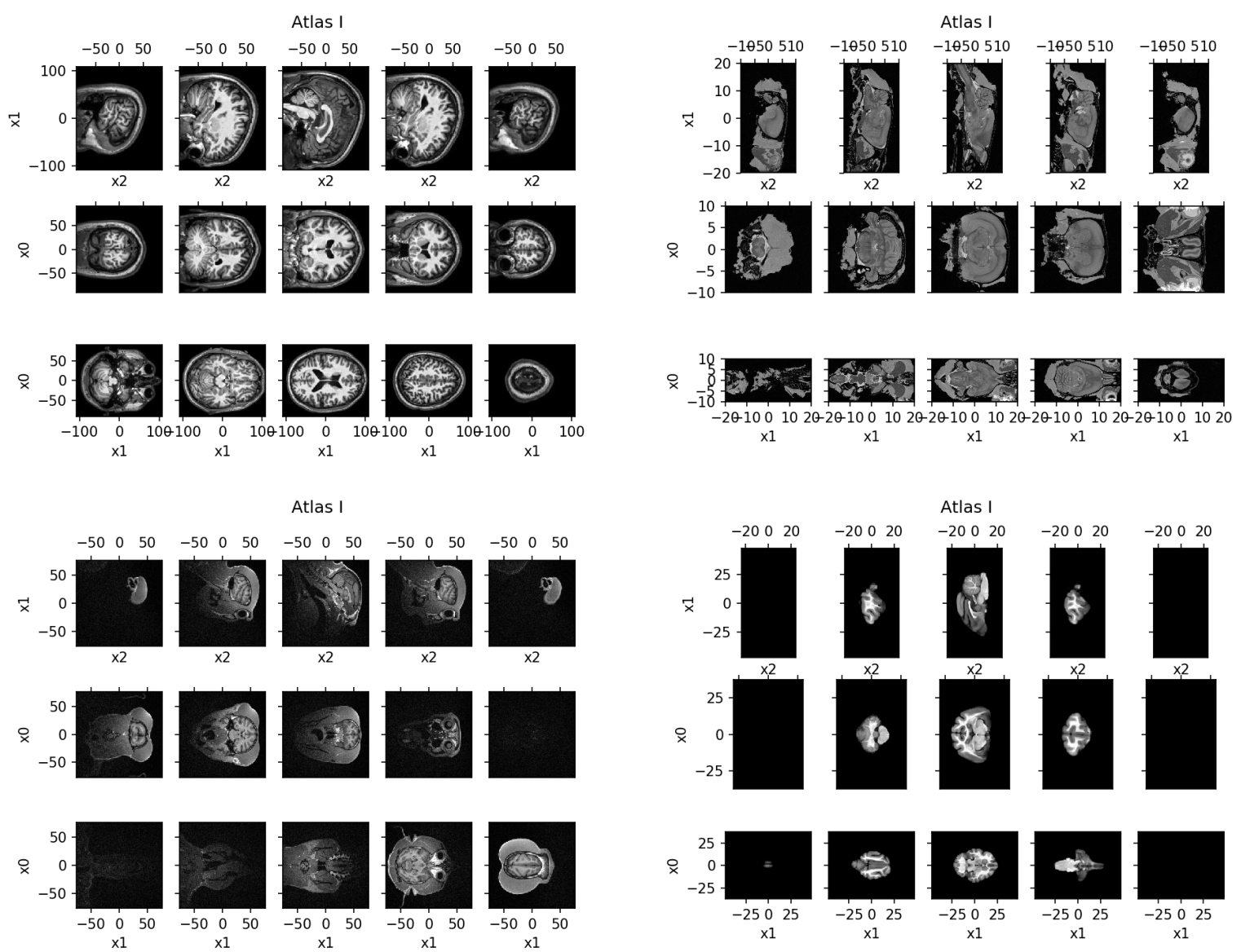

Atlas I
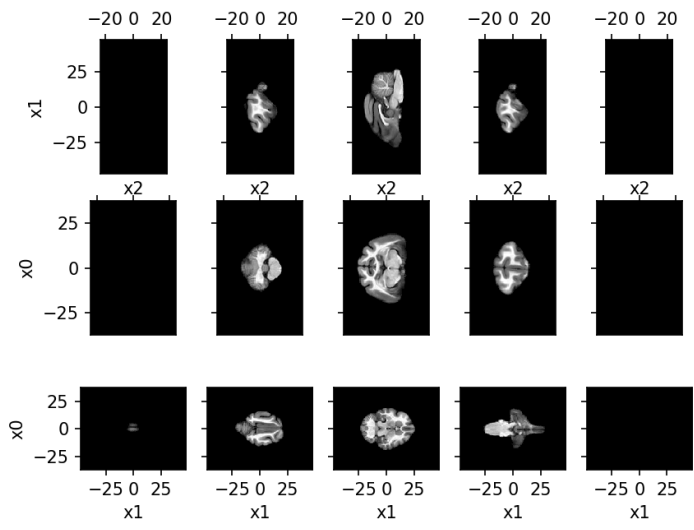

Figure 5: Additional brain atlases include human, rat, monkey, and cat.

the Computational Anatomy Science Gateway as part of the Extreme Science And Engineering Discovery Environment (XSEDE) which is supported by NSF grant ACI1548562; the Johns Hopkins University Alzheimer's Disease Research Center with NIH grant P50AG05146; the BrightFocus Foundation (www.brightfocus.org); and the Kavli Neuroscience Discovery Institute (kavlijhu.org) supported by the Kavli Foundation (www.kavlifoundation.org).

\section{REFERENCES}

[1] Brian B Avants, Charles L Epstein, Murray Grossman, and James C Gee. 2008 Symmetric diffeomorphic image registration with cross-correlation: evaluating automated labeling of elderly and neurodegenerative brain. Medical image analysis 12,1 (2008), 26-41.

[2] M Faisal Beg, Michael I Miller, Alain Trouvé, and Laurent Younes. 2005. Computing large deformation metric mappings via geodesic flows of diffeomorphisms. International journal of computer vision 61, 2 (2005), 139-157.

[3] Kwanghun Chung and Karl Deisseroth. 2013. CLARITY for mapping the nervous system. Nature Methods 10, 6 (jun 2013), 508-513. https://doi.org/10.1038/nmeth. 2481

[4] Kwanghun Chung, Jenelle Wallace, Sung-Yon Kim, Sandhiya Kalyanasundaram, Aaron S. Andalman, Thomas J. Davidson, Julie J. Mirzabekov, Kelly A. Zalocusky, Joanna Mattis, Aleksandra K. Denisin, Sally Pak, Hannah Bernstein, Charu Ramakrishnan, Logan Grosenick, Viviana Gradinaru, and Karl Deisseroth. 2013. Structural and molecular interrogation of intact biological systems. Nature 497, 7449 (apr 2013), 332-337. https://doi.org/10.1038/nature12107

[5] Arthur P Dempster, Nan M Laird, and Donald B Rubin. 1977. Maximum likelihood from incomplete data via the EM algorithm. Fournal of the royal statistical society.
Series B (methodological) (1977), 1-38.

[6] Hong Wei Dong. 2008. The Allen reference atlas: A digital color brain atlas of the C57Bl/67 male mouse. John Wiley \& Sons Inc.

[7] Paul Dupuis, Ulf Grenander, and Michael I Miller. 1998. Variational problems on flows of diffeomorphisms for image matching. Quarterly of applied mathematics (1998), 587-600.

[8] Michael N Economo, Nathan G Clack, Luke D Lavis, Charles R Gerfen, Karel Svoboda, Eugene W Myers, and Jayaram Chandrashekar. 2016. A platform for brain-wide imaging and reconstruction of individual neurons. Elife 5 (2016), e10566.

[9] Saurabh Jain, Daniel J Tward, David S Lee, Anthony Kolasny, Timothy Brown, J Tilak Ratnanather, Michael I Miller, and Laurent Younes. 2014. Computational anatomy gateway: leveraging xsede computational resources for shape analysis. In Proceedings of the 2014 Annual Conference on Extreme Science and Engineering Discovery Environment. ACM, 54.

[10] Arno Klein, Jesper Andersson, Babak A Ardekani, John Ashburner, Brian Avants, Ming-Chang Chiang, Gary E Christensen, D Louis Collins, James Gee, Pierre Hellier, et al. 2009. Evaluation of 14 nonlinear deformation algorithms applied to human brain MRI registration. Neuroimage 46, 3 (2009), 786-802.

[11] Kwame S Kutten, Nicolas Charon, Michael I Miller, J Tilak Ratnanather, Jordan Matelsky, Alexander D Baden, Kunal Lillaney, Karl Deisseroth, Li Ye, and Joshua T Vogelstein. 2017. A large deformation diffeomorphic approach to registration of CLARITY images via mutual information. In International Conference on Medical Image Computing and Computer-Assisted Intervention. Springer, 275-282.

[12] Ed S Lein, Michael J Hawrylycz, Nancy Ao, Mikael Ayres, Amy Bensinger, Amy Bernard, Andrew F Boe, Mark S Boguski, Kevin S Brockway, Emi J Byrnes, et al. 2007. Genome-wide atlas of gene expression in the adult mouse brain. Nature 445, 7124 (2007), 168.

[13] Michael P Milham, Lei Ai, Bonhwang Koo, Ting Xu, Céline Amiez, Fabien Balezeau, Mark G Baxter, Erwin LA Blezer, Thomas Brochier, Aihua Chen, et al. 
2018. An open resource for non-human primate imaging. Neuron 100, 1 (2018), 61-74.

[14] Susumu Mori, Kenichi Oishi, Andreia V Faria, and Michael I Miller. 2013. Atlasbased neuroinformatics via MRI: harnessing information from past clinical cases and quantitative image analysis for patient care. Annual review of biomedical engineering 15 (2013), 71-92.

[15] Susumu Mori, Dan Wu, Can Ceritoglu, Yue Li, Anthony Kolasny, Marc A Vaillant, Andreia V Faria, Kenichi Oishi, and Michael I Miller. 2016. MRICloud: delivering high-throughput MRI neuroinformatics as cloud-based software as a service. Computing in Science \& Engineering 18, 5 (2016), 21-35.

[16] Eszter A Papp, Trygve B Leergaard, Evan Calabrese, G Allan Johnson, and Jan G Bjaalie. 2014. Waxholm Space atlas of the Sprague Dawley rat brain. Neuroimage 97 (2014), 374-386.

[17] Nicolas Renier, Eliza L Adams, Christoph Kirst, Zhuhao Wu, Ricardo Azevedo, Johannes Kohl, Anita E Autry, Lolahon Kadiri, Kannan Umadevi Venkataraju, Yu Zhou, et al. 2016. Mapping of brain activity by automated volume analysis of immediate early genes. Cell 165, 7 (2016), 1789-1802.

[18] Nicolas Renier, Zhuhao Wu, David J. Simon, Jing Yang, Pablo Ariel, and Marc Tessier-Lavigne. 2014. iDISCO: A Simple, Rapid Method to Immunolabel Large Tissue Samples for Volume Imaging. Cell 159, 4 (nov 2014), 896-910. https: //doi.org/10.1016/j.cell.2014.10.010

[19] Andrew Staniforth and Jean Côté. 1991. Semi-Lagrangian integration schemes for atmospheric modelsâĂ ̌̌A review. Monthly weather review 119, 9 (1991), 2206-2223.

[20] Daniel Stolzberg, Carmen Wong, Blake E Butler, and Stephen G Lomber. 2017. Catlas: An magnetic resonance imaging-based three-dimensional cortical atlas and tissue probability maps for the domestic cat (felis catus). Fournal of Comparative Neurology 525, 15 (2017), 3190-3206.
[21] Daniel Tward, Michael Miller, Alain Trouve, and Laurent Younes. 2017. Parametric surface diffeomorphometry for low dimensional embeddings of dense segmentations and imagery. IEEE transactions on pattern analysis and machine intelligence 39, 6 (2017), 1195-1208.

[22] Daniel Tward, Partha Mitra, and Michael Miller. 2019. Estimating diffeomorphic mappings between templates and noisy data: Variance bounds on the estimated canonical volume form. Quart. Appl. Math. 77, 2 (2019), 467-488.

[23] Daniel Jacob Tward, Timothy Brown, Yusuke Kageyama, Jaymin Patel, Zhipeng Hou, Susumu Mori, Marilyn Albert, Juan Troncoso, and Michael Miller. 2019. Diffeomorphic registration with intensity transformation and missing data: Application to 3D digital pathology of Alzheimer's disease. BioRxiv (2019), 494005.

[24] Daniel J Tward, Can Ceritoglu, Anthony Kolasny, Gregory M Sturgeon, W Paul Segars, Michael I Miller, and J Tilak Ratnanather. 2011. Patient specific dosimetry phantoms using multichannel LDDMM of the whole body. Journal of Biomedical Imaging 2011 (2011), 3.

[25] Daniel J Tward, Brian Lee, Partha Mitra, and Michael I Miller. 2017. Performance of Image Matching in the Computational Anatomy Gateway: CPU and GPU Implementations in OpenCL. In Proceedings of the Practice and Experience in Advanced Research Computing 2017 on Sustainability, Success and Impact. ACM, 46.

[26] Joshua T Vogelstein, Eric Perlman, Benjamin Falk, Alex Baden, William Gray Roncal, Vikram Chandrashekhar, Forrest Collman, Sharmishtaa Seshamani, Jesse L Patsolic, Kunal Lillaney, et al. 2018. A community-developed open-source computational ecosystem for big neuro data. Nature methods 15, 11 (2018), 846.

[27] Jay West, J Michael Fitzpatrick, Matthew Y Wang, Benoit M Dawant, Calvin R Maurer Jr, Robert M Kessler, Robert J Maciunas, Christian Barillot, Didier Lemoine, Andre Collignon, et al. 1997. Comparison and evaluation of retrospective intermodality brain image registration techniques. fournal of computer assisted tomography 21, 4 (1997), 554-568. 